\title{
Concluding remarks for the new horizons in density functional theory Faraday Discussion
}

\author{
Andreas Savin ${ }^{1}$ \\ Laboratoire de Chimie Théorique, CNRS and Sorbonne University \\ 4 place Jussieu, 75252 Paris cedex 05, France ${ }^{\text {a) }}$
}

The present contribution tries to succintely review some progress presented during the Faraday Discussions New Horizons in density functional theory that have taken place online, 2-4 September 2020.

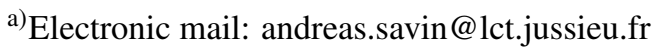




\section{WHO CARES ABOUT DFT}

50 years ago, in a conference on the numerical simulation of plasmas(!) ${ }^{1}$ Arnold C. Wahl said Some time ago, being fresh from the enthusiasm of computing Hartree-Fock (best molecular orbital) wave functions for non-trivial molecules, I gave a talk ${ }^{2}$ entitled "Hartree-Fock is Here: What Next?" and I received, from an experimentalist, a reprint request for the article "Hartree-Fock is Here - Who Cares?"

These days we discussed about "Density Functional Theory (DFT) is Here - What Next". Would somebody dare to ask today for an article "Density Functional Theory is Here - Who Cares?"? I don't think so. The position towards computational chemistry has changed in the last 50 years. A few years ago, I met an experimentalist, member of the Comite National de la Recherche Scientifique. This is the steering committee of the CNRS, the largest fundamental science agency in Europe. I asked him what he thinks about theoretical chemistry. He answered

Since we have DFT, theory competes with experiment. It became predictive.

Only DFT? Certainly not. Wave function methods also can be predictive. However, DFT computations provide good mix of accuracy and feasibility for systems of interest.

How far can we go with DFT calculations? In the nineties Walter Kohn ${ }^{3}$ made a bet. $\mathrm{He}$ predicted that, in the year 2000, DFT calculations for systems with 1000 atoms will be possible. He won the bet.

We heard from B. Civalleri ${ }^{4}$ that nowadays calculations can be performed on systems with 3000 atoms, and this routinely (on workstations with less than 100 cores, available to labs, not in huge computing centers).

We also got reinforcement from mathematicians. We used to have them tell us that we were right, when we "knew" we were right. But now, they give us new tools. M. Herbst told us how to produce bounds in our calculations ${ }^{5}$, and promises also to give us bounds on our results. I will come back to this point at the end of this manuscript.

There is also competition. From machine learning (ML). Why not? D. Cole ${ }^{6}$ showed us that models produced by ML can be (very!) fast and reliable. However, there are still aspects of machine learning that need attention. Let me enumerate some of them.

1. ML needs a heavy overhead of computations. Of course, as L. Reining pointed out ${ }^{7}$, we are used to it: in DFT we rely on uniform electron gas calculations that were done many years 
ago. However, we may ask if the input we provide to a ML model is sufficient. What if we need new information? Let me try to clarify what I mean. If we use only the electron gas calculation in DFT we have the local density approximation. However, in a solid or in a molecule, there are points having the same density contributing differently to the density functional. For example, we don't expect that the region around the center of a bond should have the same role as one the molecular surface having the same density. How can a ML model be improved? With DFT approximations introducing gradient corrections was a natural step already advocated for (it must be said, in an unsuccessful form) in the Hohenberg and Kohn paper (see, e.g., equation (48) in their $\operatorname{paper}^{8}$ ). The gradient corrections could be implemented with a conceptual, but not an important computational overhead.

2. It is not clear what data to choose to train a ML model. This, of course, is not the fault of ML, but of the existent quantum chemistry methods. With wave function methods, we don't work at the basis set limit (neither the one- or the $\mathrm{N}$-particle one). If a density functional approximation should be chosen, which one should be selected?

3. How sensitive is the ML model to the class of systems used for learning? Density functional approximations are quite robust to the change of system. It was one of features that imposed them in chemistry. For example, they could treat well not only molecules with main group elements, but also those containing transition metals. Density functionals were also used for properties for which they are not supposed to work. A typical example is using orbital energies differences to estimate band gaps, although we know that this is not a property of exact Kohn-Sham (see, e.g., ${ }^{9}$ ). Normally, ML gives out one number as an output.

Are we doomed to accept these limitations of ML? We can be certain that much can be done. L. Reining stressed that the connector approach does not prohibit the use of vectors ${ }^{7}$. J. Wetherell showed us that more flexible physics can enter into ML by using data compression to search for constraints. ${ }^{10}$. M. Gatti showed us that one can use quantities that are innovative ${ }^{11}$.

\section{CHALLENGES}

Density functional approximations have still problems. Let us discuss a few of them, as they showed up during the discussions. 


\section{Potentials}

As a rule, approximate density functionals are given as integrals over some local quantity (energy density). However, this quantity is not unique, as adding to it any function that integrates to zero does not change the value of the density functional. A truly local quantity is the Kohn-Sham potential, $v_{K S}$. The Hohenberg-Kohn theorem tells us that there is a one-to-one correspondence between it and the density. However, the density-density response function tells us that there can be many potentials that give nearly the same density (see, e.g., ${ }^{12}$ ). This might be an explanation why so many approximations work: we can have many potentials that give a similar density.

An interesting viewpoint has been presented by D. Kooi and P. Gori-Giorgi. ${ }^{13}$ She showed that one can describe exactly the leading terms of dispersion interaction by taking into account only the fragment densities, and explained that this does not violate the Hohneberg-Kohn theorem.

Nevertheless, we may want good potentials, e.g., as a starting point for the calculation of spectra. What path should we choose?

W. Yang gave arguments for a potential functional theory. ${ }^{14}$ N. Gidopoulos showed that potentials can be improved by imposing physical constraints on them. ${ }^{15} \mathrm{~N}$. Maitra showed how difficult it is to construct time-dependent potentials (nevertheless making progress with it). ${ }^{16} \mathrm{M}$. Gatti constructed models to obtain directly the spectral function by constructing models that are not based upon local, static potentials. ${ }^{11}$

A possible way to summarize is it is to say that there are other one-particle models that can be interesting (see also $\left.{ }^{7}\right)$.

\section{Spin}

Density functional theory would have never had success in thermochemistry without using spin-densities (unrestricted Kohn-Sham). However, it is not clear why they are needed in DFT. Finally, the Hohenberg-Kohn theorem tells us that for computing the energy we need only the density and the external potential. When I entered DFT, I heard people say

It is easier to get approximations if we apply a weak magnetic field. For example, for the dissociated $\mathrm{H}_{2}$ molecule, a weak magnetic field breaks the symmetry of the molecule, and stabilizes a spin-polarized solution.

Just to be clear: such a field should not be a uniform magnetic field, because this would stabilize 
the triplet, and this is not what we want. I asked myself whether the magnetic field to produce a fully polarized electron gas (the basis of almost all our approximations) is weak. For $r_{s}=$ $(3 / 4 \pi \rho)^{1 / 3}=1 \mathrm{bohr}$, it is around one atomic unit, two orders of magnitude stronger than the strongest magnetic field ever produced on Earth. ${ }^{17}$ It should not really be a surprise, as electronic excitations are needed to overcome the Pauli principle.

Different paths are being explored. C. Jacob favors using spin-state dependent exchangecorrelation functionals. ${ }^{18} \mathrm{~J}$. Neugebauer points out an alternative route to broken-symmetry calculations based on subsystems. ${ }^{19}$ Maybe one should better treat properly magnetic fields. ${ }^{20}$ Or should one give up spin-densities and concentrate on pair-densities, as done by K. Pernal ${ }^{21}$ and D. $\operatorname{Truhlar}^{22} ?$

\section{Excited states}

Initially, DFT was seen as a ground state theory. With time, it was shown that there are extensions that allow providing excitation energies, and this became one of the successes of DFT. Nevertheless, there still are questions related to it. I once discussed with a mathematician who said that from the mathematical viewpoint there is no rigorous proof for it. One day, I proposed that we have a discussion about this topic. His answer was

You want to discuss with me about a second derivative $\left[f_{x c}\right]$ of a quantity $\left[E_{x c}\right]$ for which the first derivative $\left[v_{x c}\right]$ is not well defined?

We know that we need more to have a "clean" theory. But progress is made.

P. Romaniello showed us how to get beautiful results with improved $f_{x c} \cdot{ }^{23} \mathrm{G}$. Levi developed algorithms to find saddle points (associated with some excited states). ${ }^{24}$ P.-F. Loos presented us ensembles as a way to follow. ${ }^{25} \mathrm{~W}$. Yang explained that we can go even further, and consider even changes in the number of electrons. ${ }^{14,26,27}$ D. Truhlar calculated excited states even in regions of strong state interaction, such as near conical intersections. ${ }^{22}$

\section{There is more to understand in DFT}

In 1988 T. Ziegler gave a talk in Banff. He said

We know that density functionals work. The question is why. 
He favored the explanation that density functional approximations model exchange-correlation holes. Is the Random Phase Approximation not producing models for it? J. Erhard showed us holes produced this way. ${ }^{28} \mathrm{C}$. Jacob decomposed them into symmetric and antisymmetric (singlet and triplet ${ }^{29}$ ) components and analyzed them. ${ }^{18} \mathrm{~N}$. Maitra finds a relation between why adiabatic TDDFT sometimes gives good results for dynamics in strong fields and the time-evolution of the natural occupation numbers. ${ }^{16}$ A fundamentally new approach is that proposed by K. Burke. He finds that the approximations can be justified by analyzing an asymptotic behavior $(\hbar \rightarrow 0$ keeping the chemical potential fixed). ${ }^{30}$ L. Reining explained how connector approximations generalize the path used to construct density functional approximations. ${ }^{7}$

\section{Going beyond Kohn-Sham}

Kohn-Sham had an overwhelming success. But we don't have to stay with it. J. Erhard discussed an extension of the Random Phase Approximation. ${ }^{28}$ One can consider taking into account the pair density, in particular the on-top pair density. K. Pernal combined CAS wave function with dynamic correlation obtained with density functionals; she also drew our attention to the fact that

in CASSCF calculations correlation can bring electrons together. ${ }^{21} \mathrm{D}$. Truhlar showed us a way to combine multi-reference with DFT calculations. ${ }^{22}$

\section{OUTLOOK}

We have seen that DFT is not only successful, but also has a high potential to develop. However, I would like to stress something that we are still missing in it.

We all know about J. Perdew's classification of DFT approximation according to his Jacob's ladder $^{31}$. Another well-known ladder in mystical tradition is the one of Guigo II. He wrote a book, The Ladder of Monks. Let me make an analogy between Guigo's rungs to reach God, and our steps to judge the quality of our computational results.

1. Lectio, i.e., reading. We have to be informed, we judge our results by comparing them with reference data, we use statistics. This is the method used for density functional approximations.

2. Meditatio, i.e., thinking. We know we can go further in a formal way. This is the method used in perturbation theory. 
3. Oratio, i.e., prayer. We would like to approach the exact result. This is what we do in the variational method.

4. Contemplatio. We would like to be close to the exact result. This is why we need rigorous bounds, to know how far we are from the exact solution (what M. Herbst called errors of the model ${ }^{5}$ ).

We should try to go beyond the first step, and the different contributions at this meeting have shown that we are on the good path.

\section{CONFLICTS OF INTEREST}

There are no conflicts to declare.

\section{ACKNOWLEDGEMENTS}

I would like to thank the speakers and Andrew Teale (University of Nottingham, UK) for going through the first draft of this manuscript and commenting it.

\section{REFERENCES}

${ }^{1}$ Naval Research Laboratory, Washington D.C., USA. July 1971.

${ }^{2}$ Alberta Symposium of Quantum Chemistry, University of Alberta, Canada. June 1965.

${ }^{3}$ Chemistry Nobel Prize winner 1998, considered the founding father of DFT.

${ }^{4}$ L. Donà, J. G. Brandenburg, I. J. Bush and B. Civalleri, Faraday Discuss., 2020, xx, xxx.

${ }^{5}$ M. F. Herbst, A. Levitt and E. Cancès, Faraday Discuss., 2020, xx, xxx.

${ }^{6}$ D. J. Cole, L. Mones and G. Csányi, Faraday Discuss., 2020, xx, xxx.

${ }^{7}$ A. Aouina, M. Gatti and L. Reining, Faraday Discuss., 2020, xx, xxx.

${ }^{8}$ P. Hohenberg and W. Kohn, Phys. Rev., 1964, 136, B 864.

${ }^{9}$ A. Savin, C. J. Umrigar and X. Gonze, Chem. Phys. Lett., 1998, 288, 391.

${ }^{10}$ J. Wetherell, A. Costamagna, M. Gatti and L. Reining, Faraday Discuss., 2020, xx, xxx.

${ }^{11}$ M. Vanzini, F. Sottile, I. Reshetnyak, S. Ciuchi, L. Reining and M. Gatti, Faraday Discuss., 2020, xx, xxx.

${ }^{12}$ A. Savin, F. Colonna and M. Allavena, J. Chem. Phys., 2001, 115, 6827. 
${ }^{13}$ D. P. Kooi and P. Gori-Giorgi, Faraday Discuss., 2020, xx, xxx.

${ }^{14}$ W. Yang, Faraday Discuss., 2020, xx, xxx.

${ }^{15}$ T. J. Callow, B. J. Pearce, T. Pitts, N. N. Lathiotakis, M. J. P. Hodgson and N. I. Gidopoulos, Faraday Discuss., 2020, xx, xxx.

${ }^{16}$ L. Lacombe and N. T. Maitra, Faraday Discuss., 2020, xx, xxx.

${ }^{17}$ A. Bykov, M. I. Dolotenko, N. P. Kolokolchikov and O. M. Tatsenko, Physica B: Phys. Cond. Mat., 2001, 294, 574.

${ }^{18}$ J. Brüggemann and C. R. Jacob, Faraday Discuss., 2020, xx, xxx.

${ }^{19}$ A. Massolle and J. Neugebauer, Faraday Discuss., 2020, xx, xxx.

${ }^{20}$ T. J. P. Irons and M. Wibowo discussed this topic in flash presentations of their posters at this meeting.

${ }^{21}$ K. Pernal and O. V. Gritsenko, Faraday Discuss., 2020, xx, xxx.

${ }^{22}$ J. J. Bao, C. Zhou, Z. Varga, S. Kanchanakungwankul, L. Gagliardi and D. G. Truhlar, Faraday Discuss., 2020, xx, xxx.

${ }^{23}$ S. D. Sabatino, J. A. Berger and P. Romaniello, Faraday Discuss., 2020, xx, xxx.

${ }^{24}$ G. Levi, A. V. Ivanov and H. Jónsson, Faraday Discuss., 2020, xx, xxx.

${ }^{25}$ C. Marut, B. Senjean, E. Fromager and P.-F. Loos, Faraday Discuss., 2020, xx, xxx.

${ }^{26}$ Y. Yang, H. van Aggelen and W. Yang, J. Chem. Phys., 2019, 139, 224105.

${ }^{27}$ Y. Mei, C. Li, N. Q. Su and W. Yang, J. Phys. Chem. A, 2019, 123, 666.

${ }^{28}$ J. Erhard, S. Fauser, S. Kalaß, E. Moerman, E. Trushin and A. Görling, Faraday Discuss., 2020, $\mathbf{X x}, \mathrm{XXX}$.

${ }^{29}$ R. McWeeny and W. Kutzelnigg, Intern. J. Quantum Chem., 1968, 2, 187.

${ }^{30}$ K. Burke, Faraday Discuss., 2020, xx, xxx.

${ }^{31}$ J. P. Perdew and K. Schmidt, AIP Conference Proceedings, 2001, 577, 1. 